\title{
Decreased electrogustometric taste sensitivity in patients with acquired olfactory dysfunction*
}

\author{
Pengfei Han, Marie Georgi, Mandy Cuevas, Antje Haehner, Volker Gudziol, \\ Thomas Hummel
}

Interdisciplinary Center on Smell and Taste, Department of Otorhinolaryngology, TU Dresden, Dresden, Germany
Rhinology 56: 158-165, 2018

https://doi.org/10.4193/Rhin17.186

*Received for publication:

August 18, 2017

Accepted: December 21, 2017

\begin{abstract}
Background: Cross-modal chemosensory dysfunction between olfaction and gustation is not well known.
\end{abstract}

Methodology: 180 participants were classified into three groups ( 60 with olfactory dysfunction, 60 with gustatory dysfunction and 60 healthy controls without chemosensory dysfunction). Olfactory functions were obtained with "Sniffin' Sticks"; gustatory function was measured by suprathreshold gustatory stimuli ("taste sprays") and a quasi-threshold measure of taste function ("taste strips") for five taste qualities (sweet, salty, sour, bitter and umami). "Electric taste" threshold was measured using electrogustometry (EGM). In addition, group differences in dietary behaviors were investigated with a specifically designed questionnaire.

Results: Patients with olfactory dysfunction had increased electric taste thresholds and decreased scores for the umami taste strip test as compared to healthy controls. Overall there was no major difference between patients with chemosensory dysfunction and healthy controls regarding dietary behaviors, although some patients certainly exhibited dietary problems. Importantly, patients with taste loss, but not patients with smell loss, exhibited a higher degree of depression than controls.

Conclusion: Patients with olfactory dysfunction showed decreased taste sensitivity which suggested an interaction between the chemical senses taste, trigeminal function, and olfaction. This provides the basis for including both smell and taste psychophysical assessment in clinical practices. In addition, patients with taste loss appeared to suffer most from chemosensory dysfunction.

Key words: chemosensory dysfunction, olfaction, gustation, electrogustometry, eating behavior

\section{Introduction}

Smell or taste disorders have a significant impact on the quality of daily life, including the change of dietary behaviors ${ }^{(1-3)}$. Olfaction and gustation are tightly connected ${ }^{(4,5)}$ and there is evidence that they share central processing areas, such as the insula ${ }^{(6)}$ and the orbitofrontal cortex ${ }^{(4)}$. It has been shown that olfactory impairments are accompanied by some decrease of taste function ${ }^{(7-9)}$. However, many individuals suffering from olfactory deficits who report gustatory deficits do not exhibit measurable impairments of taste function ${ }^{(10)}$. Due to the large contribution of the olfactory sense to the perception of flavor, this may be due to the confusion between gustatory impairment and a re- duced flavor experience caused by impaired retronasal olfactory function ${ }^{(11)}$. Thus, the use of objective, established tests for the assessment of gustatory function is required to investigate this topic.

Electrogustometry (EGM) is a commonly-used test based on the application of electric stimuli to the tongue. These stimuli elicit a sensation often described as metallic or sour. Studies examining electrogustometric taste thresholds showed good test-retest reliability ${ }^{(12,13)}$ although correlations with results based on chemical testing are not very strong. Previous studies have demonstrated increased EGM threshold with aging ${ }^{(14,15)}$, neurodegenerative diseases ${ }^{(16)}$, smoking ${ }^{(17)}$, tonsillectomy and 
laryngomicrosurgery ${ }^{(18)}$, drug side effects ${ }^{(19)}$, extraction of molar teeth ${ }^{(20)}$, or middle ear surgery ${ }^{(21)}$.

The main goal of this study was to investigate and compare the chemosensory functions and the possible change of dietary behavior in patients reporting olfactory deficits but not gustatory loss, as well as in patients reporting gustatory deficits but not olfactory loss. Multiple measurements were applied, including the extended 'Sniffin' Sticks' test ${ }^{(22)}$, taste spray ${ }^{(23)}$ and taste strips tests ${ }^{(24)}$, and electrogustometric taste threshold test ${ }^{(25)}$. In addition, we applied a five-taste-quality test (sweet, sour, bitter, salty, and umami) to study the use of umami taste in a clinical context. Finally, we compared dietary behaviors between patients with chemosensory dysfunctions and healthy participants.

\section{Materials and methods}

\section{Participants}

The study included 120 patients who presented to the Smell and Taste Clinic at the Department of Otorhinolaryngology at the Technical University of Dresden, complaining about smell or taste dysfunctions. There were 60 patients ( 32 females) with taste dysfunction (aged from 33.9 to 81.8 years, mean age 59.5 years), and 60 patients (43 females) with olfactory dysfunction (aged from 19.1 to 81.2 years, mean age 59.8 years). In addition, 60 healthy participants ( 38 females) aged from 23.9 to 85.9 years, mean age 56.0 years were also enrolled in this study as a control group. Table 1 provides information about subjects' sex and age, as well as the etiology of the disorder, and about the history of surgery. The main causes of chemosensory dysfunctions were idiopathic causes and infections of the upper respiratory tract, including more than $70 \%$ of the patients. There was no difference between groups in terms of age, sex distribution, body mass index (BMI) and smoking status. However, a significant difference in depression scores (obtained with the Beck Depression Inventory ${ }^{(26)}, \mathrm{BDI}$ ) was found between patients with gustatory dysfunction and healthy controls (Figure 1). According to the cutoff scores for classification of the depression severity, there were $32.8 \%$ of patients with gustatory dysfunction $(n=19)$, $24.1 \%$ of patients with olfactory dysfunction $(n=14)$, and $15.8 \%$ of control participants $(n=9)$ had mild or moderate depression (BDI score > 9) ${ }^{(27)}$.

\section{Assessment of olfactory function}

Psychophysical testing of olfactory functions was performed using the 'Sniffin' Sticks' test battery ${ }^{(22)}$. The kit comprises three subtests: 1) odor threshold for phenyl ethyl alcohol (PEA; single staircase, 3 alternative-forced choices), 2) odor discrimination (16 triplets of odors, 3 alternative-forced choice task), and 3 ) odor identification (16 common odorants, multiple forced choice from four verbal descriptors per odor). Global performance on the 'Sniffin' Sticks' test (sum of the results from threshold testing: score range 1-16; odor discrimination: score range
Table 1. Detailed description of three study groups.

\begin{tabular}{|c|c|c|c|}
\hline & $\begin{array}{l}\text { Control } \\
(n=60)\end{array}$ & $\begin{array}{c}\text { Gustatory } \\
\text { Patients } \\
(n=60)\end{array}$ & $\begin{array}{c}\text { Olfactory } \\
\text { Patients } \\
(\mathrm{n}=60)\end{array}$ \\
\hline Age (years) & $58.4 \pm 14.6$ & $59.5 \pm 14.2$ & $59.8 \pm 12.4$ \\
\hline $\operatorname{Sex}(m / f)$ & $22 / 38$ & $28 / 32$ & $17 / 43$ \\
\hline BMI & $25.6 \pm 4.2$ & $25.9 \pm 4.4$ & $26.5 \pm 3.8$ \\
\hline Smokers & $13(22.0 \%)$ & $12(21.8 \%)$ & $6(11.1 \%)$ \\
\hline Toxic exposure & 0 & 0 & 2 \\
\hline \multicolumn{4}{|l|}{ Etiology } \\
\hline Idiopathic & n.a. & $32(53.3 \%)$ & $21(35.0 \%)$ \\
\hline Postviral & n.a. & $13(21.7 \%)$ & $25(41.7 \%)$ \\
\hline Posttraumatic & n.a. & $6(10.0 \%)$ & $3(5.0 \%)$ \\
\hline Postoperative & n.a. & $8(13.3 \%)$ & $3(5.0 \%)$ \\
\hline Sinunasal & n.a. & $1(1.7 \%)$ & $8(13.3 \%)$ \\
\hline \multicolumn{4}{|l|}{ Medication } \\
\hline Antihypertensive & $24(40 \%)$ & $21(35 \%)$ & $24(40 \%)$ \\
\hline Insulin/oral antidiabetic & 0 & $9(15 \%)$ & $2(3.3 \%)$ \\
\hline Statins & $5(8.3 \%)$ & $9(15 \%)$ & $6(10 \%)$ \\
\hline Anti-rheumatics & 0 & 0 & $2(3.3 \%)$ \\
\hline Anti-depressives & 0 & $1(1.7 \%)$ & $3(5.0 \%)$ \\
\hline Thyroid drugs & $6(10 \%)$ & $4(6.7 \%)$ & $12(20 \%)$ \\
\hline Other & $8(13.3 \%)$ & $16(26.7 \%)$ & 19 (31.7\%) \\
\hline No medication & $30(50 \%)$ & $27(45 \%)$ & $20(33.3 \%)$ \\
\hline \multicolumn{4}{|l|}{ Other Diseases } \\
\hline High blood pressure & $24(40 \%)$ & $24(40 \%)$ & $24(40 \%)$ \\
\hline Diabetes & 0 & $8(13.3 \%)$ & $3(5 \%)$ \\
\hline Hypothyroidism & $6(10 \%)$ & $2(3.3 \%)$ & $6(10 \%)$ \\
\hline Neoplasia & $1(1.7 \%)$ & $2(3.3 \%)$ & $1(1.7 \%)$ \\
\hline Rheumatoid arthritis & 0 & $1(1.7 \%)$ & $2(3.3 \%)$ \\
\hline Other & $3(5 \%)$ & $4(6.7 \%)$ & $8(13.3 \%)$ \\
\hline No other diseases & $33(55 \%)$ & $30(50 \%)$ & $26(43.3 \%)$ \\
\hline \multicolumn{4}{|l|}{ Surgery histories } \\
\hline Tonsillectomy & $9(15 \%)$ & $13(21.7 \%)$ & $8(13.3 \%)$ \\
\hline Middle ear & 0 & $2(3.3 \%)$ & 0 \\
\hline Nose (Polyps, Septum) & $3(5.0 \%)$ & $5(8.3 \%)$ & $8(13.3 \%)$ \\
\hline Nasal sinuses & $1(1.7 \%)$ & $4(6.7 \%)$ & $1(1.7 \%)$ \\
\hline Teeth & $3(5.0 \%)$ & $2(3.3 \%)$ & $1(1.7 \%)$ \\
\hline Other & 0 & $4(6.7 \%)$ & $3(5.0 \%)$ \\
\hline No surgery & 45 (75\%) & $32(53.3 \%)$ & $39(65 \%)$ \\
\hline
\end{tabular}

Data shown as means \pm standard deviations and counts with proportion (in percent); * mean values not sharing a superscript are significantly different $(p<0.05)$; n.a. not applicable.

0 -16; and odor identification: score range 0 -16), designated as threshold- discrimination-identification (TDI) score range from 1 to 48) was used as the basis for classifying patients into three diagnostic groups as 1) normosmia, 2) hyposmia, and 3) anosmia. 


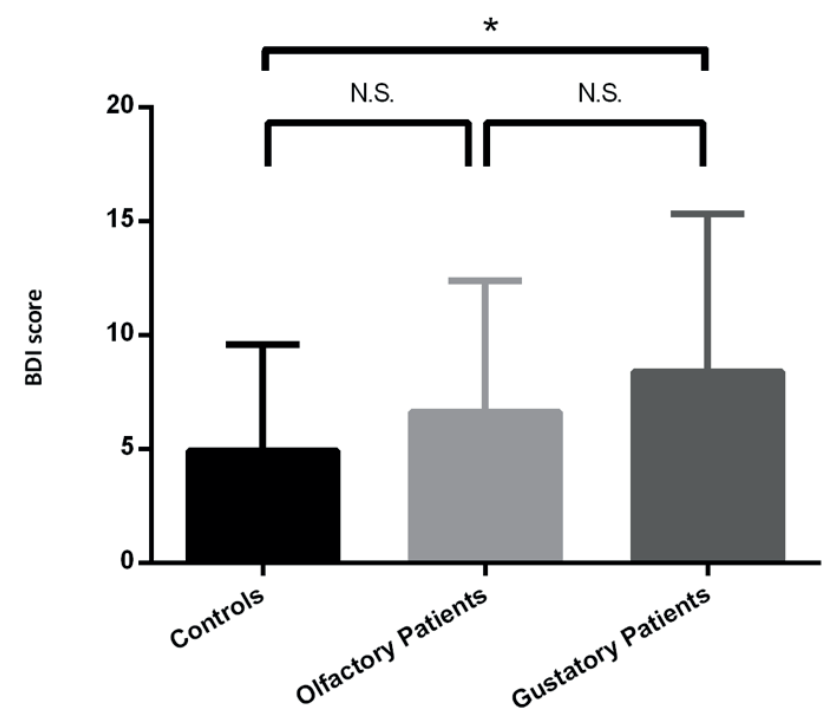

Figure 1. BDI score in controls and in patients with chemosensory dysfunctions. Bar chart shows the group mean with standard deviations and aligned dot plot for individual data; N.S. Not Significant; * significant $\mathrm{p}<0.05$.

The cut-off scores were TDI > 31 for normosmia, TDI between 15.5 to 30.5 for hyposmia, and TDI < 16.5 for anosmia ${ }^{(28)}$. During a clinical interview we also assessed the etiology of olfactory dysfunction (postinfectious, posttraumatic, sinonasal, idiopathic, congenital, toxic, or other) and the presence of parosmia and / or phantosmia.

\section{Assessment of gustatory function}

Gustatory functions were assessed by means of "taste sprays" and the "taste strips" test, well-investigated clinical tests $(29,30)$. One hour before testing, subjects were asked not to eat or drink anything except water. The "taste sprays" contain the following substances at the given concentrations (diluted in $100 \mathrm{ml}$ distilled water): sweet: $10 \mathrm{~g}$ D-saccharose, sour: $5 \mathrm{~g}$ citric acid, salty: $7.5 \mathrm{~g} \mathrm{NaCl}$, bitter: $0.025 \mathrm{~g}$ quinine hydrochloride and umami: $4 \mathrm{~g}$ monosodium glutamate ${ }^{(23,31)}$. Subjects were asked to open their mouth so that the spray could be applied (volume per spray approximately $150 \mu \mathrm{l}$ ). Afterwards they closed their mouth and moved the liquid within the mouth. The taste had to be identified as either sweet, salty, sour, bitter or umami. A score from 0 to 5 was derived from the taste spray test. Pathological results included one or more tastes incorrectly identified.

For the "taste strips" test, 20 taste-impregnated filter-paper strips were presented in a randomized order regarding taste qualities in increasing concentrations, as a whole-mouth procedure in the middle of the anterior portion of the tongue. Subjects were asked to identify the taste quality choosing one of five possible answers on a form (sweet, sour, salty, bitter, umami). Before assessment of each taste strip, the mouth was rinsed with water. The taste score was the number of correctly identified taste strips. The following concentrations were used for the taste strips: sweet: $0.4,0.2,0.1,0.05 \mathrm{~g} / \mathrm{ml}$ sucrose; sour: $0.3,0.165,0.09$, $0.05 \mathrm{~g} / \mathrm{ml}$ citric acid; salty: $0.25,0.1,0.04,0.016 \mathrm{~g} / \mathrm{ml}$ sodium chloride; bitter: $0.006,0.0024,0.0009,0.0004 \mathrm{~g} / \mathrm{ml}$ quinine hydrochloride; umami: $0.25,0.1,0.04,0.016 \mathrm{~g} / \mathrm{ml}$ monosodium glutamate. The total score for taste strip testing ranged from 0 to 20. Taste Strips have been shown to be useful in clinical practice (30): They exhibit several advantages, such as short time needed for testing, good reproducibility of the results, the possibility to test each side of the tongue separately, and a very long shelf life. In addition, the etiology of gustatory dysfunction (postinfectious, posttraumatic, idiopathic, congenital, toxic, or other) and the presence of parageusia and/or phantogeusia were assessed.

\section{Assessment of electrogustometric taste sensitivity} The electric taste threshold was measured using electrogustometric stimulation. The electric stimulus was applied with a bi-polar electrode (round surface of $0.79 \mathrm{~cm}^{2}$ ) using an electrogustometer (TR06, Rion, Tokyo, Japan). The electrode was placed on two anterior regions of the tongue (tongue tip, and edge), separately for the left and right sides. Stimuli were applied in increasing strengths ( $2 \mathrm{~dB}$ steps). Stimuli of $0.5 \mathrm{~s}$ duration were applied unilaterally, starting at $6 \mathrm{~dB}(1.5 \mathrm{~mA})$ up to $40 \mathrm{~dB}$, until the subject indicated that the applied stimulus had been perceived. If the subject did not perceive the $40 \mathrm{~dB}$ stimulus, a $1 \mathrm{~mA}(50 \mathrm{~dB}$ ) stimulus was applied. If no sensation was perceived, the highest possible value $(50 \mathrm{~dB})$ was entered into the analysis. The stimulation frequency was $2 \mathrm{~Hz}$.

\section{Dietary evaluation}

For the investigation of changes for dietary behavior following the change of chemosensory function, a specially designed questionnaire was used. The questions were related to changes in weight (weight gain or weight loss), meals per day and time spent on each meal, eating related to mental or physical stress, eating habits such as emptying of food plates although full, facilitated eating with visual food cues, frequent food craving, and alcohol consumption. Answers varied according to the type of questions asked. For example, patients were asked to refer to standardized answers for questions related to stress related eating ("more,"'no change," "less") and for alcohol consumption ("none,"' "occasionally," "regularly"); binary answers (either 'yes' or 'no') were applied to questions about weight loss/ weight gain, empty food plate, visual facilitated eating habits and food craving; open and non-structured answers were applied to other questions such as the meals per day and meal time. Since this questionnaire was applied in a study for the first time, psychometric characteristics of this instrument need to be addressed in future investigations.

\section{Statistical analysis}

The Statistical Package for the Social Sciences (SPSS vs. 21.0; 


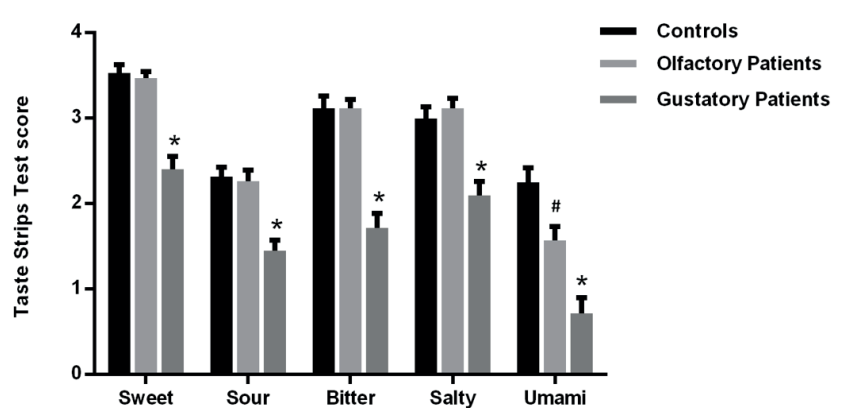

Figure 2. "Taste Strips” gustatory functions for five basic taste qualities in controls and in patients with olfactory or gustatory dysfunctions. * Gustatory patients had significantly reduced score for each specific taste as compared to controls and olfactory patients ( $p<0.05$ ); \# Umami taste strips test score significantly reduced in olfactory patients as compared to controls $(p<0.05)$.

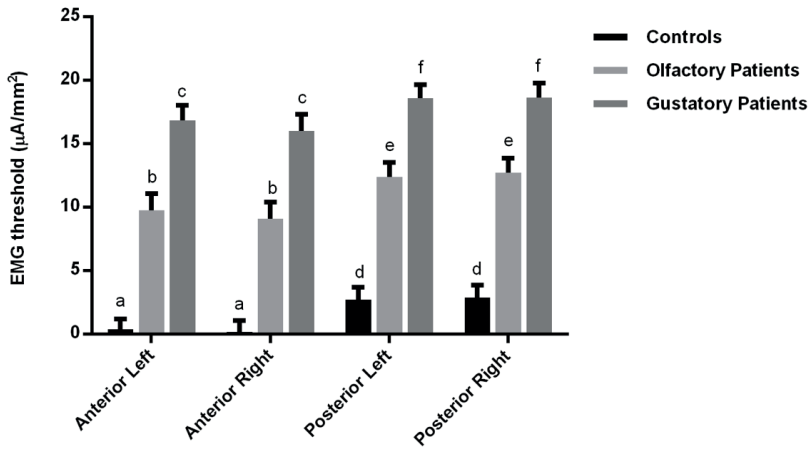

Figure 3. Regional electrogustometric taste threshold $(\mu \mathrm{A} / \mathrm{mm} 2)$ in healthy controls and patients with chemosensory disorders. Anterior left, anterior right, posterior left, and posterior right indicate the quadrants of the tongue. Bars show the mean values with SEM. Different superscript letters ( $a, b, c, d, e, f)$ indicate significant differences (paired t-test, $p<0.05)$.
SPSS Inc., Chicago, IL, USA) and Graphpad Prism (version 6; GraphPad Software, Inc.) was used for data analyses and graphical plot. Descriptive and parametric interferential statistical tests (e.g., X2 tests, Mann-Whitney tests, Pearson correlations, KruskalWallis tests) were applied wherever appropriate. The a-level was adjusted to 0.05 . Data were presented as Mean with standard deviation or mean with standard error as indicated.

\section{Results}

\section{Chemosensory functions}

Compared to controls, significantly reduced TDI olfactory test scores were found for patients with olfactory dysfunctions (18.7 \pm 7.8 v.s. $29.8 \pm 2.9, \mathrm{p}<0.001)$, but not for patients with gustatory dysfunctions $(26.3 \pm 10.2$ vs. $29.8 \pm 2.9, p>0.05)$. Patents with gustatory dysfunctions exhibited significantly lower taste strips scores for all five taste qualities five basic tastes in comparison to healthy controls and olfactory patients. Moreover, patients with olfactory dysfunctions showed lower umami taste score compared to healthy controls ( $p<0.05$, Figure 2 ), but no difference was seen for the other four tastes ( $p>0.05$, Figure 2). For the taste spray test, patients with gustatory dysfunctions had significantly lower scores for all taste qualities $(p<0.05)$, while the scores for patients with olfactory dysfunctions were not significantly different from controls $(p>0.05)$. In addition, the symptom "dry mouth" was not more frequent in patients with gustatory dysfunctions ( 24 out of $60,40 \%$ ) and patients with olfactory dysfunctions ( 16 out of $60,26.7 \%)(x 2$ test $p=$ 0.087).

\section{EGM taste sensitivity}

At the four recording positions, the electrogustometric taste thresholds were significantly higher in patients with olfactory dysfunctions as compared to healthy controls. Patients with taste dysfunctions exhibited further increased electrogustometric thresholds compared to patients with olfactory dysfunctions (Figure 3). In addition, the EMG threshold was lower at the frontal part as compared to the posterior part of the tongue (Figure 3).

Significant correlations were found between EGM threshold (averaged across 4 positions) and taste test scores in patients with gustatory dysfunctions (Spearman correlations $r=0.29$, $p=0.024$ sweet 'taste strips' score; $r=-0.384, r=0.003$ for combined taste spray test score; and $r=-0.349, p=0.007$ for umami 'taste spray' score). Significant correlations were also found between EGM threshold (averaged across 4 positions) and taste test scores in healthy controls (Spearman correlations $r=-0.342$, $p=0.008$ for sour 'taste spray' score; and $r=-0.273, p=0.037$ for bitter 'taste spray' score). There was no significant correlation between EGM threshold and taste test scores among patients with olfactory disorders $(p>0.1)$.

\section{Patients with parageusia / phantogeusia}

Among the patients with gustatory dysfunction ( $n=60), 28$ patients (46.7\%) were diagnosed with parageusia, and 21 patients (35\%) with phantogeusia; 15 patients (25\%) were diagnosed with having both parageusia and phantogeusia. There were 34 out of 60 (56.7\%) patients with gustatory dysfunctions who were diagnosed with either parageusia or phantogeusia or both; and this subgroup of patients $(n=34)$ had significantly higher taste strips test score for 'bitterness' compared to patients with gustatory dysfunction but without parageusia or phantogeusia $(p<0.001)$. There was also a trend for taste strips score for 'salty' ( $p=0.059$, Table 2 ). In addition the combined taste strips test score was also higher in the subgroup of patients compared to other patients in the group ( $p<0.05$, Table 2 ). The TDI score for this subgroup (TDI $=30.8 \pm 7.2$ ) of patients with either parageu- 
Table 2. Taste and smell test scores between subgroups of patients with gustatory dysfunctions.

\begin{tabular}{|lccc|}
\hline & $\begin{array}{c}\text { Patients without } \\
\text { parageusia } \\
\text { or phantogeusia } \\
(\mathbf{n}=\mathbf{3 4})\end{array}$ & $\begin{array}{c}\text { Patients with } \\
\text { parageusia } \\
\text { or phantogeusia } \\
(\mathbf{n}=\mathbf{2 6})\end{array}$ & $\begin{array}{c}\mathbf{P} \\
\text { value }\end{array}$ \\
\hline TS bitter & $0.9 \pm 0.8$ & $2.3 \pm 1.3$ & $<0.001$ \\
\hline TS total & $7.6 \pm 2.8$ & $9.0 \pm 2.9$ & $<0.05$ \\
\hline TS salty & $1.8 \pm 1.1$ & $2.4 \pm 1.3$ & $=0.059$ \\
\hline TDI score & $20.5 \pm 10.6$ & $30.8 \pm 7.2$ & $<0.001$
\end{tabular}

Data are shown as means \pm standard deviations; TS, Taste Strips test; TDI score, the combined olfactory test score of 'Sniffin' Sticks' test.

sia and/or phantogeusia were significantly higher than the rest of patients (TDI $=20.5 \pm 10.6$ ) (Table 2).

\section{Patients with parosmia / phantosmia}

Among the patients with olfactory dysfunction, 10 patients had parosmia, and 7 patients had phantosmia. There were $23.3 \%$ of olfactory patients $(n=14)$ who had parosmia or phantosmia or both. TDI score of this patient subgroup $(n=14)$ was not different from the other patients with olfactory dysfunctions (TDI score, Mann-Whitney Test $Z=-1.66, p=0.10)$. However, patients with parosmia / phantosmia had significantly higher EGM threshold (were less sensitive) compared to the other olfactory patients without parosmia or phantosmia (averaged EGM threshold = $17.6 \pm 6.5$ for patients with parosmia/ phantosmia; $8.4 \pm 7.6$ for patients without parosmia / phantosmia, Mann-Whitney Test Z = $-3.57, \mathrm{p}<0.001)$.

\section{Dietary behaviors}

Alcohol consumption was found different between study groups, with patients with olfactory or gustatory dysfunctions consuming alcohol less frequently than healthy controls. No significant difference was observed in terms of eating habits (Table 3). $26.7 \%$ (16 out of 60 ) patients with olfactory dysfunction, and $16.7 \%$ ( 10 out of 60 ) patients with gustatory dysfunction reported chemosensory-disorder-related weight loss, and this was not different between the two patient groups ( $\mathrm{X} 2=1.77, \mathrm{df}=1$, $p>0.1)$. Olfactory or gustatory dysfunction was not associated with changes of the number of meals per day compared to healthy controls; neither did the averaged time spent on each meal.

\section{Discussion}

The current study investigated the olfactory and gustatory functions as well as the dietary behaviors in patients with smell or taste loss. One important finding was that patients with olfactory dysfunction exhibited significantly increased EGM taste
Table 3. Eating habits of healthy controls and patients with gustatory or olfactory dysfunctions.

\begin{tabular}{|c|c|c|c|}
\hline & $\begin{array}{l}\text { Control } \\
(n=56)\end{array}$ & $\begin{array}{c}\text { Gustatory } \\
\text { Patients } \\
(n=54)\end{array}$ & $\begin{array}{c}\text { Olfactory } \\
\text { Patients } \\
(n=42)\end{array}$ \\
\hline $\begin{array}{l}\text { Empty food plate alt- } \\
\text { hough full }\end{array}$ & $20(35.7 \%)$ & $18(33.3 \%)$ & $12(28.6 \%)$ \\
\hline $\begin{array}{l}\text { Facilitated eating by } \\
\text { visual cue }\end{array}$ & $17(30.4 \%)$ & $10(19.2 \%)$ & $7(16.7 \%)$ \\
\hline Frequent food craving & $12(21.4 \%)$ & $10(19.2 \%)$ & $15(35.7 \%)$ \\
\hline More eating under stress & $8(14.3 \%)$ & $6(11.1 \%)$ & $5(11.9 \%)$ \\
\hline Less eating under stress & $20(35.7 \%)$ & $18(33.3 \%)$ & $17(40.5 \%)$ \\
\hline $\begin{array}{l}\text { No change of eating } \\
\text { under stress }\end{array}$ & $26(46.4 \%)$ & $29(53.7 \%)$ & $20(47.6 \%)$ \\
\hline
\end{tabular}

Data are shown as the number of subjects and their proportion of the group (in percentage); There is no significant difference between study groups in all the eating habits ( $x 2$ tests $p>0.1$ for all).

thresholds, suggesting impaired gustatory function among patients with olfactory loss. EGM threshold has been reported to be negatively correlated with the number of fungiform papillae (32, 33). Previous studies have demonstrated increased EGM threshold with aging ${ }^{(14,15)}$ or smoking ${ }^{(17)}$. While some studies favored the electric taste as a gustatory phenomenon ${ }^{(34-36)}$, others failed to show a significant correlation between electric and common chemical tastes ${ }^{(37-39)}$, signifying the unique nature of electric taste. In addition, electric stimulation may be contaminated by trigeminal activation ${ }^{(40)}$. Brain mapping studies with source localization analyses showed activations in the primary gustatory cortex and the somatosensory cortex with electrical stimuli applied to the tongue ${ }^{(35,41,42)}$. Previous studies have found that patients with olfactory loss also exhibited a decreased trigeminal sensitivity ${ }^{(43,44)}$. Taking into account the peripheral and central interactions between the trigeminal and gustatory systems, the increased EMG threshold may also indicate a negative impact of olfactory dysfunction on the trigeminal-taste interactions. In addition, olfaction and gustation share common central processing areas which may account for the cross-modal chemosensory changes ${ }^{(6,45,46)}$, which provides the neuropathic basis for the bidirectional influence between the two ${ }^{(4,7)}$. For example, people with congenital olfactory impairments were worse in taste identification and exhibited reduced taste-induced brain activations ${ }^{(8)}$. However, in the present study the "taste strips" test results indicated no significant taste dysfunction in those patients with olfactory loss, except for umami taste. Future studies with larger sample size are needed to investigate the umami taste impairments in patients with olfactory dysfunction. In addition, it seems that patients with olfactory dysfunction preserved normal suprathreshold taste function a measured 
with "taste sprays" test.

Although the patients with gustatory dysfunction did not show olfaction impairment as indicated by their 'Sniffin Sticks' test score (TDI score), a subgroup of 36 patients without parageusia or phantogeusia had significantly lower TDI score (average TDI $=20.5 \pm 10.6$ ). The mechanism behind this phenomenon is not clear. One possibility is that the qualitative dysfunctions of olfaction or gustation may have an additional impact on the chemosensory interactions. Future studies with larger sample size need to address this hypothesis. In addition, compared to patients with parageusia or phantogeusia, the subgroup of patients without parageusia or phantogeusia had significantly lower scores for overall taste and bitter scores of the 'taste strips' test. This result is in accordance with a previous study showing that the salty and bitter-salty mix sensations were the most frequently reported in patients with parageusia or phantogeusia ${ }^{(47)}$.

It needs to be noted that EGM is limited to investigations on particular taste qualities, and EGM taste thresholds fail to predict suprathreshold function at levels experienced in everyday life. However, its specific clinical role may be in the discrimination of ageusia/hypogeusia or the characterization of localized taste loss in the 4 different quadrants of the tongue which is difficult to reach with pipettes or filter papers ${ }^{(48)}$. In fact, human taste function is complex and it appears that no single measure is capable of being used as a definitive marker of overall taste function ${ }^{(49,50)}$. Our data also suggest the potential usefulness of a combination of taste measurements in clinical tests, for patients with chemosensory dysfunctions ${ }^{(51)}$.

The patients with gustatory dysfunctions had significantly higher BDI scores compared to controls, while the patients with olfactory dysfunctions did not. There were nearly one thrid of patients with gustatory dysfunction (32.8\%) had a BDI scor over 10 , which fall in the range of mild to moderate depression. In addition, the average BDI (mean score $=8.4$ ) for those patients was close to the cutoff point ${ }^{(26)}$. While previous studies have suggested olfaction as an early marker for depression ${ }^{(52)}$, the results from this study may draw attention on the gustatory patients in clinical practices. Future investigations are warranted before any conclusive claim can be made for the relationship between gustatory dysfunction and depression.

There was no significant weight change for the patients' groups involved in the current investigation. Previous studies reported inconsistent findings of weight changes due to chemosensory dysfunctions $^{(3,10,53-55)}$. It has also been reported that weight change (either gain or loss of body weight) might be associated with the type of chemosensory dysfunctions ${ }^{(54)}$. In addition, the current study did not reveal significant differences between smell- or taste- loss patients and healthy participants in terms of dietary behaviors. Previous studies had reported various changes of dietary behaviors in patients with gustatory or olfactory loss ${ }^{(3,54,55)}$. Those studies yielded different pictures of dietary problems associated with chemosensory disorders. However, overall the results suggest that at least some individuals may exhibit clinically significant dietary and weight changes. Therefore it seems to be highly important to identify individuals at nutritional risk.

The use of questionnaires to investigate dietary behaviors in patients with chemosensory deficits does not allow the study on potential changes in food perception (e.g. flavor pleasantness and intensity, flavor/taste preferences, flavor induced appetite, etc.). It has been shown that most complaints of taste loss reflect olfactory rather than gustatory dysfunction ${ }^{(10)}$. This is due to the common confusion of taste and smell in people and the critical role of olfaction in determining the appreciation of flavor in foods and beverages. In addition, the decrement in food enjoyment among patients with chemosensory dysfunctions seems to be extremely high (from $60 \%$ to $90 \%$ of the patients) (2, 3). Therefore, it is necessary for future studies to investigate the impact of chemosensory dysfunctions on actual food perception (e.g. taste, smell, flavor, texture etc.) using real food items ${ }^{(7)}$.

\section{Conclusion}

In conclusion, results from the current study showed decreased gustatory sensitivity among patients with olfactory dysfunction which underlines the role of chemosensory interactions. In addition, patients with gustatory disorders were more depressed than patients with olfactory loss and controls. Routine chemosensory investigation should include assessment of taste and smell.

\section{Authorship contribution}

PH: data analysis and write up; MG: draft of study, conduct of experiments, help with writeup; MC: conduct of experiments, help with writeup; $\mathrm{AH}$ : conduct of experiments, help with writeup; VG: conduct of experiments, help with writeup; TH: draft of study, conduct of experiments, help with write up.

\section{Conflict of interest}

None.

\section{References}

1. Hummel T, Nordin S. Olfactory disorders and their consequences for quality of life. Acta Oto-Laryngologica. 2009; 125: 116-21.

2. Croy I, Nordin S, Hummel T. Olfactory disor- ders and quality of life--an updated review. Chem Senses. 2014; 39: 185-94.

3. Mattes RD, Cowart BJ, Schiavo MA, Arnold C, Garrison B, Kare MR, et al. Dietary evaluation of patients with smell and/or taste disorders. Am J Clin Nutr. 1990; 51: 233-40. 4. Frasnelli J, Collignon O, Voss P, Lepore F. Crossmodal plasticity in sensory loss. In: Green A, Chapman C, Kalaska J, Lepore F, editors. Progress in brain research. 191. 1st 
Ed. ed. Amsterdam: Elsevier; 2011; 233-49.

5. Kollndorfer K, Kowalczyk K, Frasnelli J, Hoche E, Unger E, Mueller CA, et al. Same same but different. Different trigeminal chemoreceptors share the same central pathway. PLoS One. 2015; 10: e0121091.

6. Lundstrom JN, Boesveldt S, Albrecht J. Central Processing of the Chemical Senses: an Overview. ACS Chem Neurosci. 2011; 2 5-16.

7. Landis BN, Scheibe M, Weber C, Berger R, Bramerson A, Bende M, et al. Chemosensory interaction: acquired olfactory impairment is associated with decreased taste function. J Neurol. 2010; 257: 1303-8.

8. Gagnon L, Vestergaard M, Madsen K, Karstensen HG, Siebner H, Tommerup N et al. Neural correlates of taste perception in congenital olfactory impairment. Neuropsychologia. 2014; 62: 297-305.

9. Gudziol H, Rahneberg K, Burkert S. Anosmics are more poorly able to taste than normal persons. Laryngo-rhinootologie. 2007; 86: 640-3.

10. Deems DA, Doty RL, Settle RG, Moore-Gillon $V$, Shaman P, Mester AF, et al. Smell and taste disorders, a study of 750 patients from the University of Pennsylvania Smell and Taste Center. Arch Otolaryngol Head Neck Surg. 1991; 117: 519-28.

11. Rozin P. "Taste-smell confusions" and the duality of the olfactory sense. Percept Psychophys. 1982; 31: 397-401.

12. Murphy C, Quinonez C, Nordin S. Reliability and validity of electrogustometry and its application to young and elderly persons. Chem Senses. 1995; 20: 499-503.

13. Stillman JA, Morton RP, Goldsmith D. Automated electrogustometry: a new paradigm for the estimation of taste detection thresholds. Clin Otolaryngol Allied Sci. 2000; 25: $120-5$.

14. Pavlidis P, Gouveris H, Anogeianaki A, Koutsonikolas D, Anogianakis G, Kekes G. Age-related changes in electrogustometry thresholds, tongue tip vascularization, density, and form of the fungiform papillae in humans. Chem Senses. 2013; 38: 35-43.

15. Saito $T$, Ito $T$, Ito $Y$, Manabe $Y$, Sano $K$. Comparison of fungiform taste-bud distribution among age groups using confocal laser scanning microscopy in vivo in combination with gustatory function. Eur J Oral Sci. 2016; 124: 135-40.

16. Shah M, Deeb J, Fernando M, Noyce A, Visentin E, Findley LJ, et al. Abnormality of taste and smell in Parkinson's disease. Parkinsonism Relat Disord. 2009; 15: 232-7.

17. Khan AM, Narayanan VS, Puttabuddi JH, Chengappa R, Ambaldhage VK, Naik P, et al. Comparison of Taste Threshold in Smokers and Non-Smokers Using Electrogustometry and Fungiform Papillae Count: A Case Control Study. J Clin Diagn Res. 2016; 10: ZC101-5.

18. Tomofuji S, Sakagami M, Kushida K, Terada T, Mori H, Kakibuchi M. Taste disturbance after tonsillectomy and laryngomicrosurgery. Auris Nasus Larynx. 2005; 32: 381-6.
19. Tsuruoka S, Wakaumi M, Araki N, loka T, Sugimoto K, Fujimura A. Comparative study of taste disturbance by losartan and perindopril in healthy volunteers. J Clin Pharmacol. 2005; 45: 1319-23.

20. Morton RP, Hay KD, Goldsmith DB, Stillman JA. Patterns of sensory recovery in the lingual nerve after surgical trauma. N Z Dent J. 2005; 101: 53-7.

21. Nin T, Sakagami M, Sone-Okunaka M, Muto T, Mishiro Y, Fukazawa K. Taste function after section of chorda tympani nerve in middle ear surgery. Auris Nasus Larynx. 2006; 33: 13-7.

22. Hummel T, Sekinger B, Wolf SR, Pauli E, Kobal G. 'Sniffin' Sticks': Olfactory performance assessed by the combined testing of odor identification, odor discrimination and olfactory threshold. Chem Senses. 1997; 22: 39-52.

23. Hummel T, Hummel C, Welge-Luessen A. Assessment of olfaction and gustation. In: Welge-Luessen A, Hummel T, editors. Management of smell and taste disorders: a practical guide for clinicians 1st ed. Stuttgart: Thieme; 2013; 58-75.

24. Gudziol H, Hummel T. Normative values for the assessment of gustatory function using liquid tastants. Acta oto-laryngologica. 2007; 127: 658-61.

25. Krarup B. Electro-gustometry: a method for clinical taste examinations. Acta Otolaryngol. 1958; 49: 294-305.

26. Beck AT, Steer RA, Carbin MG. Psychometric properties of the Beck Depression Inventory: Twenty-five years of evaluation. Clin Psychol Rev. 1988; 8: 77-100.

27. Kendall PC, Hollon SD, Beck AT, Hammen $\mathrm{CL}$, Ingram RE. Issues and Recommendations Regarding Use of the Beck Depression Inventory. Cognit Ther Res. 1987; 11: 289-99.

28. Hummel T, Kobal G, Gudziol H, Mackay-Sim A. Normative data for the "Sniffin' Sticks" including tests of odor identification, odor discrimination, and olfactory thresholds: an upgrade based on a group of more than 3,000 subjects. Eur Arch Otorhinolaryngol. 2007; 264: 237-43.

29. Mueller C, Kallert S, Renner B, Stiassny K, Temmel AFP, Hummel T, et al. Quantitative assessment of gustatory function in a clinical context using impregnated "taste strips". Rhinology. 2003; 41: 2-6.

30. Landis BN, Welge-Luessen A, Bramerson A Bende M, Mueller CA, Nordin S, et al. "Taste Strips" - a rapid, lateralized, gustatory bedside identification test based on impregnated filter papers. J Neurol. 2009; 256: 242-8.

31. Mueller CA, Pintscher K, Renner B. Clinical test of gustatory function including umami taste. Ann Otol Rhinol Laryngol. 2011; 120: 358-62.

32. Saito $T$, Ito $T$, Ito $Y$, Yamada $T$, Okamoto $M$, Manabe Y. Gustatory Dysfunction and Decreased Number of Fungiform Taste Buds in Patients With Chronic Otitis Media With Cholesteatoma. Ann Otol Rhinol Laryngol. 2016; 125: 704-9.
33. Miller SL, Mirza N, Doty RL. Electrogustometric thresholds: Relationship to anterior tongue locus, area of stimulation, and number of fungiform papillae. Physiol Behav. 2002; 75: 753-7.

34. Stillman JA. Revisiting the Relationship Between Electrogustometry and Sour Taste Perception. Open Otorhinolaryngol J. 2008; 2: 57-9.

35. Ohla K, Toepel U, le Coutre J, Hudry J. Electrical neuroimaging reveals intensitydependent activation of human cortical gustatory and somatosensory areas by electric taste. Biol Psychol. 2010; 85: 446-55.

36. Barry MA, Gatenby JC, Zeiger JD, Gore JC Hemispheric dominance of cortical activity evoked by focal electrogustatory stimuli. Chem Senses. 2001; 26: 471-82.

37. Stevens DA, Baker D, Cutroni E, Frey A, Pugh D, Lawless HT. A direct comparison of the taste of electrical and chemical stimuli. Chem Senses. 2008; 33: 405-13.

38. Lawless HT, Stevens DA, Chapman KW Kurtz A. Metallic taste from electrical and chemical stimulation. Chem Senses. 2005: 30: 185-94.

39. Ellegard EK, Goldsmith D, Hay KD, Stillman JA, Morton RP. Studies on the relationship between electrogustometry and sour taste perception. Auris Nasus Larynx. 2007; 34: 477-80.

40. Stillman JA, P. MR, D. HK, Z A, D G. Electrogustometry: stengths, weaknesses, and clinical evidence of stimulus boundaries. Clin Otolaryngol Allied Sci. 2003; 28: 406-10.

41. Ohla K, Hudry J, le Coutre J. The cortical chronometry of electrogustatory eventrelated potentials. Brain Topogr. 2009; 22: 73-82.

42. Yamamoto C, Takehara S, Morikawa K, Nakagawa S, Yamaguchi M, Iwaki S, et al. Magnetoencephalographic study of cortical activity evoked by electrogustatory stimuli. Chem Senses. 2003; 28: 245-51.

43. Frasnelli J, Hummel T. Interactions between the chemical senses: trigeminal function in patients with olfactory loss. Int J Psychophysiol. 2007; 65: 177-81.

44. Hummel T, Barz S, Lotsch J, Roscher S, Kettenmann B, Kobal G. Loss of olfactory function leads to a decrease of trigeminal sensitivity. Chem Senses. 1996; 21: 75-9.

45. Small DM, Prescott J. Odor/taste integration and the perception of flavor. Exp Brain Res. 2005; 166: 345-57.

46. Shepherd GM. Smell images and the flavour system in the human brain. Nature. 2006; 444: 316-21

47. Fark T, Hummel C, Hahner A, Nin T, Hummel T. Characteristics of taste disorders. Eur Arch Otorhinolaryngol. 2013; 270: 1855-60.

48. Walliczek U, Negoias S, Hahner A, Hummel T. Assessment of Chemosensory Function Using "Sniffin' Sticks", Taste Strips, Taste Sprays, and Retronasal Olfactory Tests. Curr Pharm Des. 2016; 22: 2245-52.

49. Webb J, Bolhuis DP, Cicerale S, Hayes JE, Keast R. The Relationships Between 
Common Measurements of Taste Function. Chemosens Percept. 2015; 8: 11-8.

50. Snyder DJ, Fast K, Bartoshuk LM. Valid comparisons of suprathreshold sensations. J Conscious Stud. 2004; 11: 96-112.

51. Hummel $T$, Whitcroft $K L$, Andrews $P$ Altundag A, Cinghi C, Costanzo RM, et al. Position paper on olfactory dysfunction. Rhinology. 2017 Jun 17. doi: 10.4193/ Rhin $16.248 \mathrm{~V}$

52. Croy I, Hummel T. Olfaction as a marker for depression. J Neurol. 2017; 264: 631-8.

53. Ferris AM, Duffy VB. Effect of olfactory deficits on nutritional status: does age predict persons at risk? Ann N Y Acad Sci. 1989; 561: 113-23.

54. Mattes RD, Cowart BJ. Dietary assessment of patients with chemosensory disorders. J Am Diet Assoc. 1994; 94: 50-6.

55. Aschenbrenner K, Hummel C, Teszmer K Krone F, Ishimaru T, Seo HS, et al. The influence of olfactory loss on dietary behaviors. Laryngoscope. 2008; 118: 135-44.

\section{Pengfei Han}

Interdisciplinary Center on Smell and

Taste

Department of Otorhinolaryngology

TU Dresden

Fetscherstrasse 74

01307 Dresden

Germany 